CrossMark $\leftarrow$ click for updates

Cite this: Nanoscale, 2014, 6, 10486

Received 6th June 2014

Accepted 30th June 2014

DOI: $10.1039 / c 4 n r 03124 \mathrm{e}$

www.rsc.org/nanoscale

\title{
Synthetic micro/nanomotors in drug delivery
}

\author{
Wei Gao and Joseph Wang*
}

Nanomachines offer considerable promise for the treatment of diseases. The ability of man-made nanomotors to rapidly deliver therapeutic payloads to their target destination represents a novel nanomedicine approach. Synthetic nanomotors, based on a multitude of propulsion mechanisms, have been developed over the past decade toward diverse biomedical applications. In this review article, we journey from the use of chemically powered drug-delivery nanovehicles to externally actuated (fuel-free) drug-delivery nanomachine platforms, and conclude with future prospects and challenges for such practical propelling drug-delivery systems. As future micro/nanomachines become more powerful and functional, these tiny devices are expected to perform more demanding biomedical tasks and benefit different drug delivery applications.

\section{Introduction}

\section{Drug delivery needs}

Drug delivery technology is an area of enormous importance to health care, and aims at addressing the deficiencies of conventional means for administering drugs., ${ }^{1,2}$ The need for the development of innovative technologies to improve the delivery of therapeutic agents in the body has been widely recognized. Considerable research efforts have thus been directed over the past 15 years to the development of targeted drug delivery systems aimed at preventing and treating debilitating diseases. ${ }^{3}$

Department of Nanoengineering, University of California, San Diego, La Jolla, CA, 92093, USA.E-mail:josephwang@ucsd.edu
Such delivery platforms promise to address several key issues, including low therapeutic efficacy and significant negative side effects by delivering a drug where the medication is needed, while sparing healthy parts of the body. ${ }^{4,5}$ New technologies are also needed for delivering therapeutic agents to areas of the body that are currently inaccessible to current delivery methods and for improving tissue penetration.

\section{Towards nanomedicine}

Nanotechnology-enabled drug delivery systems have received considerable interest owing to their major impact on the treatment of diseases. ${ }^{6,7}$ The promise of such nanotechnologybased systems is to deliver a drug selectively to the target tissues and cells with increased efficacy while reducing side effects. ${ }^{3} \mathrm{~A}$

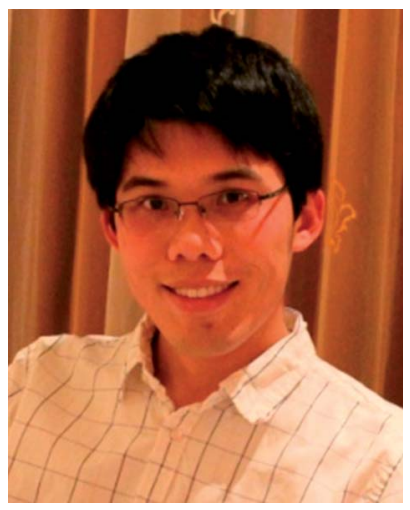

Wei Gao received his $P h D$ in Chemical Engineering at University of California, San Diego in 2014, where he worked under the supervision of Professor Joseph Wang on synthetic micro/nanomachines. He has received Jacobs Fellowship of UC San Diego, 2012 HHMI International Student Research fellowship, 2012 Chinese Government Award for Outstanding Self-financed Students Abroad and MRS Graduate Student Silver Award (2013 and 2014). He is currently a postdoctoral fellow at the University of California, Berkeley. His research interests include nanomaterials, naonomachines, electrochemistry, MEMS/NEMS and nanomedicine.

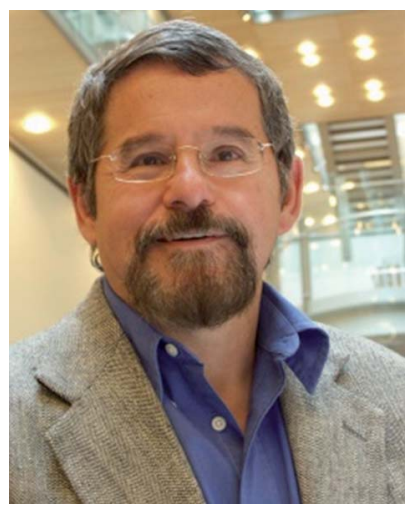

Joseph Wang is a Distinguished Professor and Chair of NanoEngineering at University of California San Diego (UCSD), USA. After holding a Regents Professor position at NMSU he moved to ASU where he served as the Director of the Center for Bioelectronics and Biosensors (Biodesign Institute). He joined the UCSD NanoEngineering Dept. in 2008. He also serves as the Chief Editor of Electroanalysis. The research interests of Dr Wang include the development of nanomotors and nanoactuators, bioelectronics and biosensors, and "smart" wearable sensor systems. He has authored over 900 research papers, 10 books, 12 patents, and 35 chapters (H index 104). 
wide range of nanomaterial-based systems have thus been developed for drug delivery applications, including liposomes, polymeric nanoparticles, dendrimers, polymersomes, nanoemulsions. ${ }^{1,2}$ Significant progress has been made towards improving the therapeutic efficacy of drug-loaded nanoparticles, including extended drug systemic circulation lifetime, improved the solubility of poorly water-soluble drugs, codelivery of two or more drugs or of therapeutic and imaging agents within the same particle (i.e., "theranostics"), and controlled or triggered release of the encapsulated drugs. While functionalizing the particle surface with targeting ligands can result in accumulation around tumors, off-targeting (and the corresponding adverse effect) remains a major challenge. Another challenge of common drug delivery vehicles is the lack of power necessary for penetrating tissue and cellular barriers. Deep tissue penetration and cellular interaction thus represents another important goal. To achieve such active targeting and deliver payloads to predetermined locations in the body, future generation drug delivery vehicles may need to incorporate propulsion and navigation capabilities. Nanoshuttles, precisely guided by a physician, have the potential to transport therapeutic agents directly to diseased tissues, and to access nearly every site of the human body through blood vessels, independent of the blood flow, thereby improving the therapeutic efficacy and reducing systemic side effects of highly toxic drugs.

\section{Nanomotors for drug delivery}

The use of nanomotors to power nanomachines is currently a research area of tremendous activity due to a wide range of potential applications. ${ }^{8-17}$ Synthetic nanomotors, based on a multitude of propulsion mechanisms, have thus been developed over the past decade..$^{8-10,18}$ While most of this attention has been given to chemically powered catalytic micromotors, ${ }^{19-23}$ many important applications (particularly in vivo biomedical ones) require the elimination of the fuel requirements toward biocompatible propulsion mechanisms. Efforts in this direction have led to the fuel-free locomotion of magnetically driven nanoswimmers, ${ }^{24-28}$ or acoustically propelled devices..$^{29-31}$ Major advances in nanomotor technology, including the design of powerful multifunctional machines, advanced motion control and cargo towing capabilities, have facilitated different biomedical applications ranging from cell sorting ${ }^{32}$ to DNA hybridization. ${ }^{33}$ The substantial progress towards using functionalized nanomotors for efficient cargo transport and release paves the way to their drug-delivery applications. The targeted delivery of therapeutic cargoes represents a major future application of synthetic micromotors. ${ }^{16}$ Such nanomachine-based drug delivery systems are highly attractive platforms for efficient delivery of therapeutic payloads to targeted sites, and could address some of the obstacles of current drug delivery systems. Unfortunately, there is no comprehensive review published on using synthetic micro/nanomachines for drug delivery platforms.

In this review we highlight recent research efforts aimed at developing man-made nanomachines for drug delivery applications and give an outlook on current challenges and emerging trends. Table 1 summarizes recent progress of drug delivery based on diverse nanomotors powered by different mechanisms. These new drug-delivery nanoshuttles are discussed in the following sections, along with related opportunities and challenges.

\section{Catalytically powered micro/ nanomotors for targeted drug delivery}

Catalytically powered micro/nanoscale motors rely on the catalytic decomposition of a solution-borne fuel, usually hydrogen peroxide, on a platinum surface. ${ }^{19-23}$ Such fuel-driven motors possess a relatively high power essential for performing different biomedical tasks involving cargo towing. ${ }^{46}$ This force is reflected by a remarkable speed that can exceed 1000 bodylengths s ${ }^{-1} .{ }^{47}$ Motion control is another important requirement for targeted drug delivery. The directionality of catalytic micro/ nanoscale motors can be readily controlled (commonly via magnetic guidance) and their speed can be regulated using different stimuli. ${ }^{48}$ Tremendous progress has been made on cargo-carrying catalytic nanomotors based on different loading and unloading mechanisms. ${ }^{49}$ These advances have facilitated efforts aimed at using a variety of catalytic nanomotors (based on wire, sphere or open-tube configurations) for targeted drug delivery.

Wang, Zhang and coworkers demonstrated the first example of using man-made micromotors for the transport and release of drugs. ${ }^{34}$ This pioneering study illustrated that catalytic nanowire shuttles can readily pickup drug-loaded poly D,L-lactic-co-glycolic acid (PLGA) particles and liposomes and transport them over predetermined routes towards target destinations. Powerful alloy or CNT-based nanowire motors have been used to increase the force necessary to transport 'heavy' therapeutic cargos. These nickel-containing motors captured the iron-oxide encapsulated PLGA and liposome drug nanocarriers through magnetic interactions. The nanomotors can thus pickup, transport and release varying sized drug carriers towards predetermined destinations. Fig. 1 displays scheme (A) and time-lapse images (B) of such dynamic capture, transport and release of doxorubicin (DOX)-loaded PLGA nanoparticles using a catalytic $\mathrm{Ni} /\left(\mathrm{Au}_{50} / \mathrm{Ag}_{50}\right) / \mathrm{Ni} / \mathrm{Pt}$ nanowire motor. In comparison with the PLGA particles, transport of the drug-loaded liposome is relatively slower due to its large size.

Wu et al. reported the preparation of a well-defined multilayer tubular polymeric nanomotor through the nanoporous template-assisted layer-by-layer (LbL) assembly. ${ }^{35}$ Platinum nanoparticles (Pt NPs), assembled into the inner surface of LbLassembled nanotubes, provided the catalytic decomposition of the hydrogen peroxide fuel essential for the bubble propulsion. These nanorockets can serve as autonomous motors as well as smart cargos, performing drug loading, targeted transportation and remote-controlled release in the vicinity of cells and tissues. The fluorescent anticancer drug doxorubicin was loaded onto the nanorockets through encapsulation. An ultrasound (US) 
Table 1 Recent progress on drug delivery using micro/nanomotors based on a multitude of propulsion mechanisms and designs

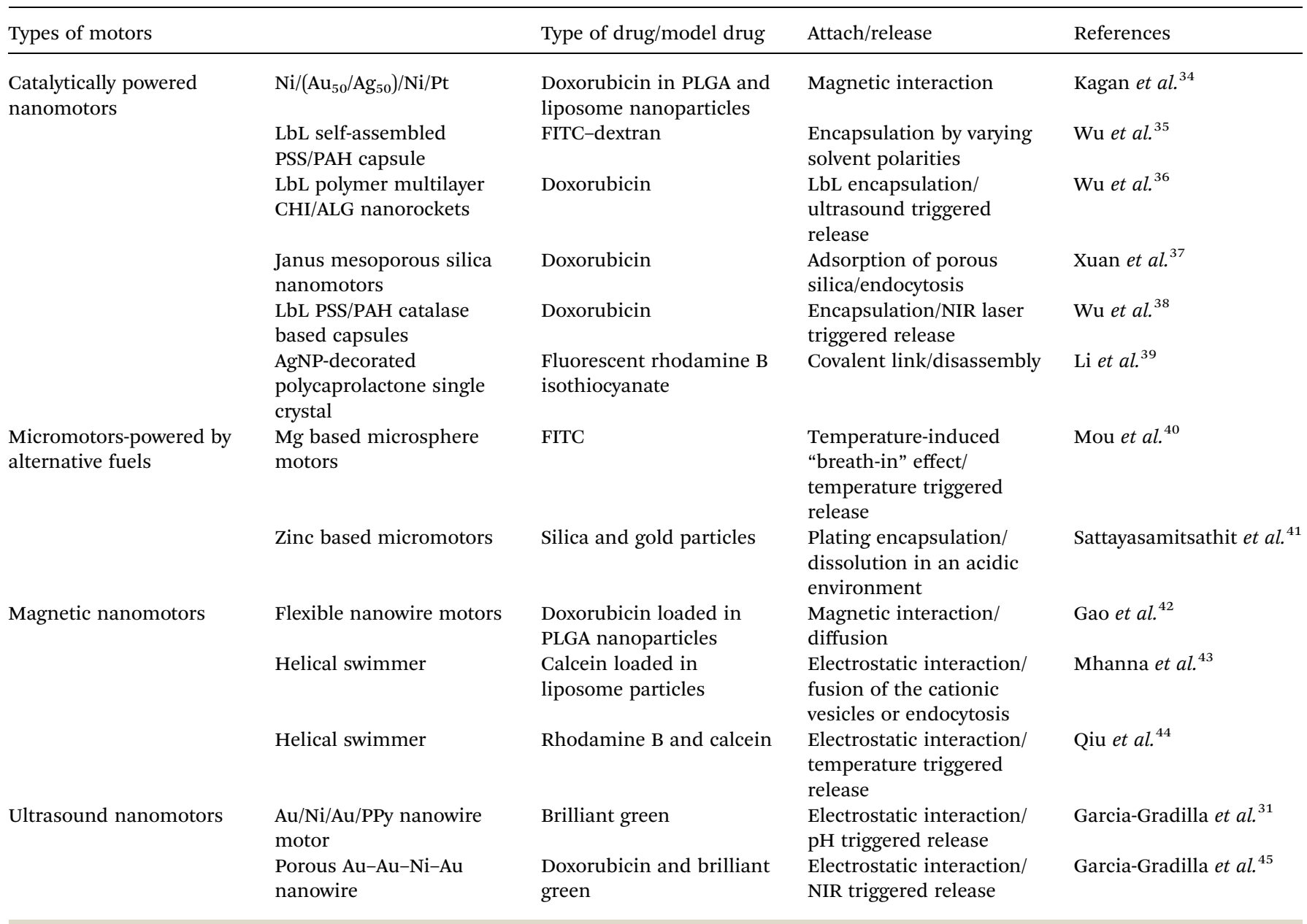

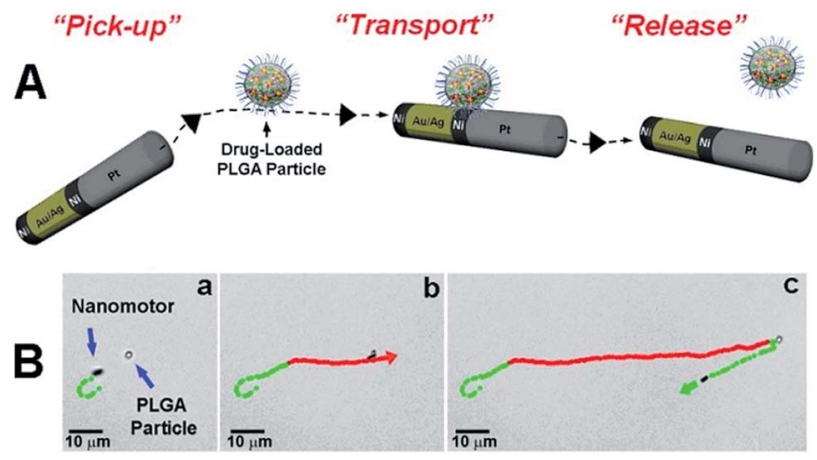

Fig. 1 Transport and release of PLGA drug carriers by catalytic nanowire motors. Schematic (A) and microscopic time lapse-images (B) depicting the dynamic pick-up (a), transport (b), and release (c) of drug-loaded PLGA particles using a nanoshuttle. (Reproduced from ref. 34, Wiley 2010.)

field was used to trigger the breakage of the outer shell of the LbL assembled polyelectrolyte multilayer microcapsules and release the encapsulated drugs. The images in Fig. 2 display (alginate (ALG)/chitosan (CHI) $)_{4}-\mathrm{DOX}-(\mathrm{ALG} / \mathrm{CHI})_{2}-\mathrm{Fe}_{3} \mathrm{O}_{4}-(\mathrm{CHI} /$ $\mathrm{ALG})_{14}-\mathrm{Pt}$ NP nanorockets attached to the outer surface of HeLa
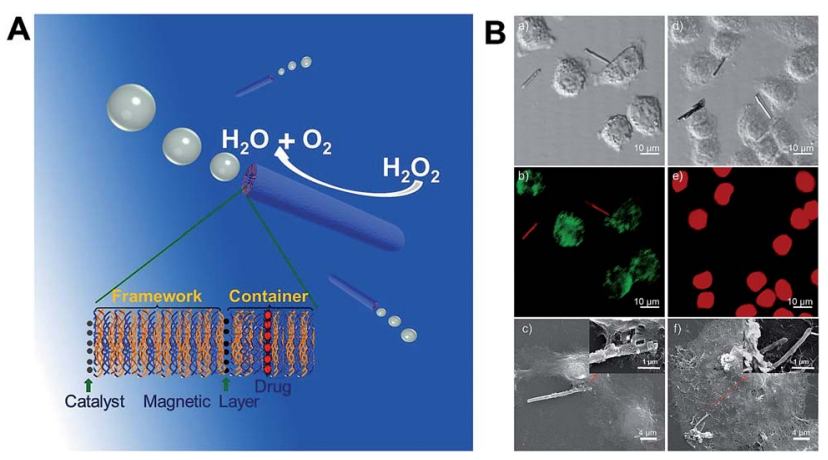

Fig. 2 (A) Self-assembled polymer multilayer nanorockets based on a template-assisted layer-by-layer (LBL) technique can propel chemically in the presence of a hydrogen peroxide fuel. These motors can perform drug loading, targeted transportation, and triggered drug release by an external physical stimulus in a controlled manner. (B) The DIC ( $a$ and $d$ ), the corresponding CLSM ( $b$ and e), and SEM images ( $c$ and f) of a $(\mathrm{CHI} / \mathrm{ALG})_{4}-\mathrm{DOX}-(\mathrm{ALG} / \mathrm{CHI})_{2}-\mathrm{Fe}_{3} \mathrm{O}_{4}-(\mathrm{CHI} / \mathrm{ALG})_{14}-\mathrm{PtNP}$ nanorocket before $(\mathrm{a}-\mathrm{C})$ and after $(\mathrm{d}-\mathrm{f})$ ultrasound treatment in vitro and continuous cultivation of the HeLa cells for $3 \mathrm{~h}$. (Reproduced from ref. 35, Wiley 2013.) 
cancer cells. Differential interference contrast (DIC), confocal laser scanning microscopy (CLSM), scanning electron microscopy (SEM), and atomic force microscopy (AFM) images show the corresponding results before and after ultrasonic treatment. In comparison with the image before ultrasonic treatment (Fig. 2Bb), the red fluorescence from DOX in the CHI/ALG multilayer nearly disappeared (Fig. 2Be), indicating that most of DOX molecules have been released through the ultrasound irradiation. Similar concepts were also demonstrated in LBL capsule based micromotors. Wu et al. reported also the use of dendritic Pt-nanoparticles or catalase-modified polyelectrolyte capsules as micromotors as well as carriers for drug delivery. ${ }^{36,38}$ Fluorescein isothiocyanate-dextran (FITC-dextran) model drugs or doxorubicin have been loaded onto these microcapsules by encapsulation.

Another recent work from the same Chinese group described a new self-propelled Janus silica nanomotor which can also serve as the drug carrier for intracellular drug delivery. ${ }^{37}$ As illustrated in Fig. 3, this peroxide-propelled catalytic Janus nanomotor is based on mesoporous silica nanoparticles (MSNs) with chromium/platinum metallic caps. MSNs offer an exceptionally high surface area, which enables the loading of diverse cargoes. The DOX drug was loaded into mesoporous pore channels of Janus MSN motors by physical adsorption, and then covered with an egg phosphatidylcholine (PC) bilayer. Intracellular localization and drug release experiments in vitro have indicated that the amount of Janus MSN nanomotors entering the cells is more than control MSNs with the same culture time and particle concentrations, meanwhile anticancer drug doxorubicin hydrochloride loaded in Janus MSNs can be slowly released in the cells by biodegradation of the lipid bilayers.

Other recent studies hold considerable promise for a variety of biomedical applications. For example, Sanchez et al. reported the use of self-propelled rolled-up microtubes to drill and embed themselves into biomaterials such as cells which can be potentially used to address the endosome escape challenge and deliver the drug or gene inside the cell. ${ }^{50}$ Another study by $\mathrm{Wu}$ et al. ${ }^{51}$ demonstrated that by taking advantages of photothermal effects, PtNP-modified polyelectrolyte multilayer microtube engines can be used for targeted recognition and subsequent killing of cancer cells.

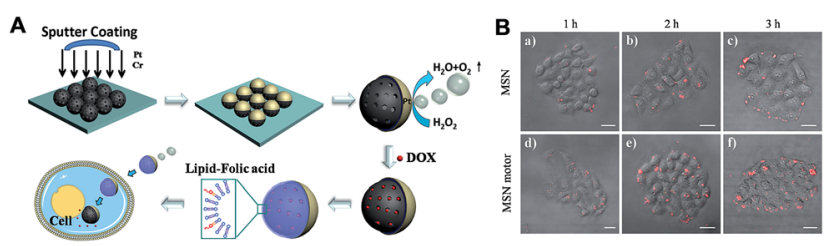

Fig. 3 (A) Synthetic procedure for the preparation of Janus MSN nanomotors, as well as subsequent drug loading, lipid bilayer functionalization, transportation, and drug release. (B) DOX release from egg PC modified Janus MSN nanomotors inside HeLa cells following: (a) 0, (b) 1, (c) 2, and (d) $3 \mathrm{~h}$. The images are overlays of fluorescence and DIC channels. Scale bars, $10 \mathrm{~mm}$. (Reproduced from ref. 37, Wiley 2014.)

\section{Micromotors powered by alternative fuels for drug delivery}

Although major progress has been made on drug delivery based on catalytic micro/nanomotors, current reliance on the common hydrogen peroxide fuel greatly hinders practical biomedical applications of catalytic micromotors. In particular, in vivo drug delivery applications would require the use of body fluids or their constituents as the powering fuel. Recent efforts have been directed at expanding the scope of fuels for synthetic nanomotors by exploring the use of natural biofluids as the fuel source, thus obviating the need for external chemical fuels. ${ }^{52-56}$ For example, Gao et al. described an acid-driven polyaniline/ zinc microtube rocket that can propel autonomously and efficiently in gastric acid, and thus can be operated in the stomach environment. ${ }^{52}$ Reports of the use of glucose-powered enzymebased carbon swimmers are also promising in this respect, although the propulsion mechanism is based on current flow, and was shown to diminish at high salt concentrations. ${ }^{57}$

Of particular interest for practical drug delivery applications are recently developed water-driven micromotors that utilize the magnesium-water reaction for the propulsion. ${ }^{55,56}$ These magnesium-based Janus microsphere motors consist of biodegradable magnesium microparticles coated with a gold or platinum patch. Such water-driven micromotors utilize macrogalvanic corrosion and chloride pitting corrosion processes to generate hydrogen bubbles that propel the microparticles. This eliminates the need for external fuels and offers efficient propulsion in untreated high-salt aquatic media which is particularly attractive for in vivo drug delivery applications. Another water-driven micromotor explores Janus particles based on the Al-Ga alloy for efficient hydrogen bubble propulsion through a process called 'liquid metal enbrittlement', ${ }^{54}$ but lacks the biocompatibility of magnesium-based micromotors. Additional efforts should be devoted to extending the lifetime of these water-powered micromotors, along with proper surface functionalization, for addressing the requirements of practical drug delivery.

Mou et al. described recently a biocompatible drug-loaded magnesium-based $\mathrm{Mg} / \mathrm{Pt}$-poly( $N$-isopropylacrylamide) (PNIPAM) Janus micromotor (Fig. 4). ${ }^{40}$ Such water-powered micromotors display an efficient autonomous motion in simulated body fluid (SBF) or blood plasma without any additives or fuels. It offers also attractive capabilities of loading, transporting and delivering drug molecules by taking advantages of the partial surface-attached thermoresponsive PNIPAM hydrogel layers. The drug releasing process from the micromotor can be controlled by the environmental temperature due to the 'squeeze' effect. Hemolysis assay has suggested that such micromotors and their autonomous motion have a negligible influence on the red blood cells (RBCs, highly hemocompatible) and are friendly to organisms.

Combination therapy offers several distinct advantages for disease treatment (e.g., high efficiency, synergistic effects and reversal of drug resistance) compared to normal single drug therapy. ${ }^{6,58}$ Such combinatorial drug delivery may require new 

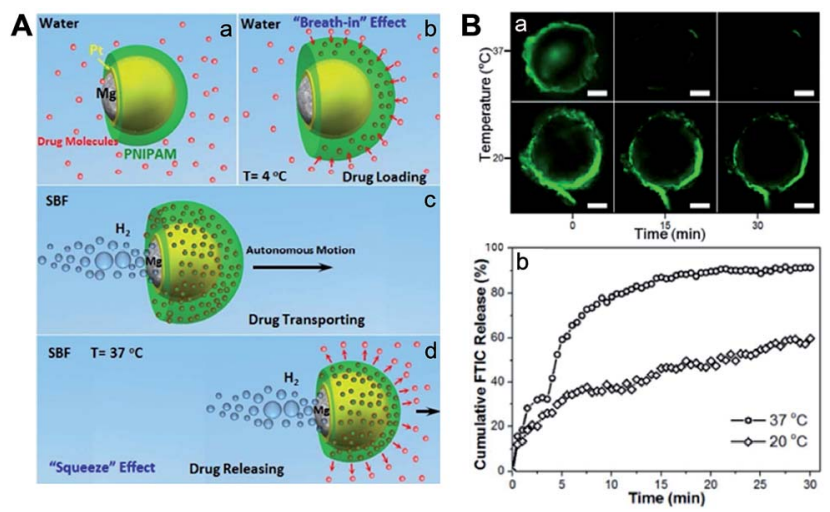

Fig. 4 (A) Schematic demonstration of the drug (a and b) loading, (c) transporting, and (d) releasing behaviors of the Mg/Pt-PNIPAM Janus micromotors. (B) Fluorescent images representing the drug release from the Mg/Pt-PNIPAM Janus microsphere motor (a) and the normalized average cumulative drug release profiles (b) at 20 and $37{ }^{\circ} \mathrm{C}$ versus time. Scale bars: $10 \mu \mathrm{m}$. (Reproduced from ref. 40, American Chemical Society 2014.)

vehicles that enable co-encapsulation of different drugs, active targeting, and/or temporally controlled release. A recent work from our laboratory illustrated a fully loaded multi-cargo zinc based micromotor which can be potentially used for combinatorial delivery (Fig. 5). ${ }^{41}$ Such an acid-powered bubble-propelled micromotor possesses several distinct functions for potential biomedical use. These include remarkably high loading capacity, combinatorial delivery of a multitude of cargoes, autonomous release of encapsulated payloads, and selfdestruction. Such multifunctional zinc-based micromotors, prepared by dual-templating nanofabrication, can move rapidly in an acidic environment and transport the fully loaded cargoes with a speed of $110 \mu \mathrm{m} \mathrm{s}^{-1}$. As the zinc body is oxidized and dissolved by the acid fuel, the different cargoes are released autonomously and the motors are self-destroyed, leaving

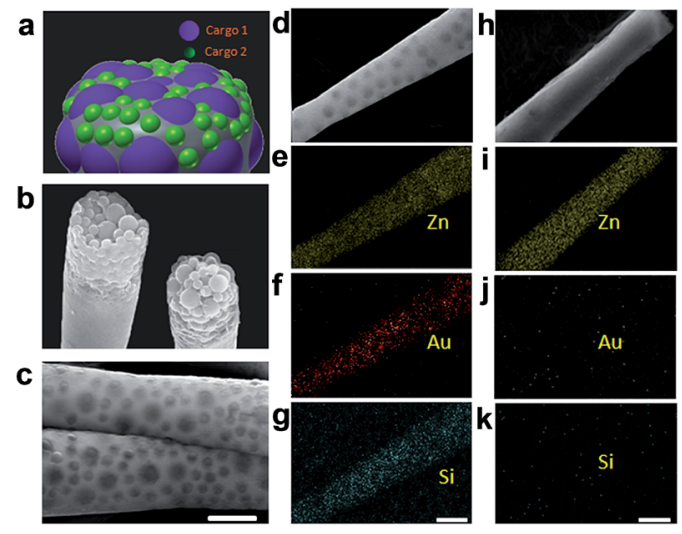

Fig. 5 SEM images and energy-dispersive $X$-ray spectroscopy (EDX) analysis of fully loaded dual-cargo $\mathrm{Zn}$ micromotors towards combinatorial therapy. (a-d) Zn micromotors encapsulated with $500 \mathrm{~nm}$ $\mathrm{SiO}_{2}$ particles. (e-h) control $\mathrm{Zn}$ micromotors without the $\mathrm{SiO}_{2}$ particles. Scale bar, $0.5 \mu \mathrm{m}$ (b) and (f), $1 \mu \mathrm{m}$ (a) and (e), and $2 \mu \mathrm{m}$ (c), (d), (g) and (h). (Reproduced from ref. 41, Wiley 2014.) behind no harmful chemical agents. This attractive concept can be readily expanded to simultaneous encapsulation of a wide variety of payloads, possessing different biomedical functions such as therapy, diagnostics, and imaging, hence opening up new drug delivery opportunities.

\section{Magnetic micro/nanomotors for drug delivery}

To address the limitations of fuel-driven micromotors and enhance biocompatibility, several groups have been exploring fuel-free micro/nanomachine propulsion mechanisms, including the utilization of magnetic, ${ }^{24-28}$ electrical, ${ }^{59-61}$ optical $^{62}$ or ultrasound ${ }^{29-31}$ fields. Magnetically driven nanomotors, inspired by nature swimming microorganisms, are particularly promising for use in a variety of in vivo biomedical applications. ${ }^{12}$ Such micromotors can swim under externally applied magnetic fields in various biofluids, and perform complex maneuvers while obviating fuel requirements. Magnetic actuation is suitable for in vivo applications since the required field-strengths are harmless to humans. Ghosh et al. reported recently the first successful "voyage" of magnetic nanomotors, based on conformal ferrite coatings, in human blood. ${ }^{63}$ Such magnetic "nanovoyagers" were shown to be cytocompatible with mouse myoblast cells. Magnetically actuated micromachines are thus currently being explored extensively as promising platforms for controlled in vivo drug delivery applications.

Gao et al. reported the first example of directed delivery of drug-loaded magnetic polymeric particles using magnetic nanoswimmers. ${ }^{42}$ The fundamental mechanism of the cargotowing ability and the hydrodynamic features of these flexible nanowire motors have been discussed. The effect of the cargo size on the swimming performance was evaluated experimentally and compared to a theoretical model, emphasizing the interplay between hydrodynamic drag forces and boundary actuation. Potential applications of these cargo-towing nanoswimmers were demonstrated using the directed delivery of

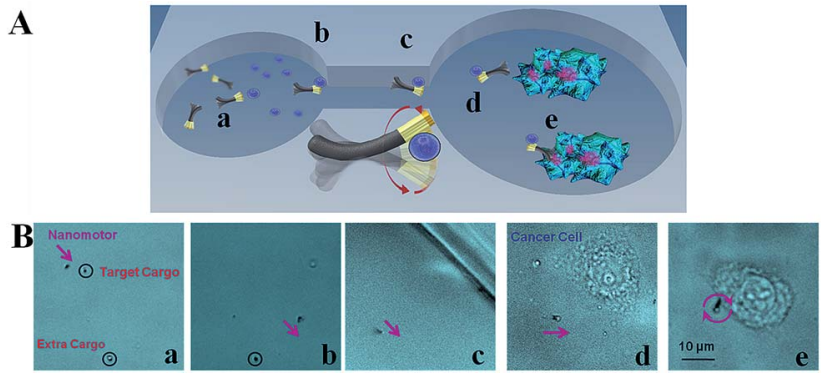

Fig. 6 Drug delivery to HeLa cells using flexible magnetic nanoswimmers in cell-culture media. Scheme (A) and microscopic timelapsed images (B) depicting the process as a flexible magnetic $\mathrm{Ni}-\mathrm{Ag}$ nanowire motor (a) capturing the doxorubicin-loaded magnetic poly( $D, L$-lactic-co-glycolic acid) (PLGA) particle in the loading reservoir (b), transporting it through the microchannel (c), approaching the target cell (d), sticking onto the target cell, and releasing the drug (e). (Reproduced from ref. 42, Wiley 2014.) 
drug-loaded microparticles to HeLa cancer cells in biological media. Fig. 6 illustrates the schematic and experimental results of using the magnetic nanowire swimmers for transporting of target iron oxide/doxorubicin-encapsulated PLGA particles through a microchannel from the pick-up zone to the release microwell.

As was illustrated by Nelson's group in ETH, magnetically actuated helical micromachines hold considerable promise for diverse biomedical applications. ${ }^{\mathbf{6 4 , 6 5}}$ The Swiss team developed the artificial bacterial flagella (ABFs) which are capable of performing precise $3 \mathrm{D}$ navigation in fluids under rotating magnetic fields and are harmless to cells and tissues. ${ }^{28}$ They reported the successful functionalization of titanium-coated ABFs with temperature-sensitive dipalmitoyl phosphatidylcholine (DPPC)based liposomes. The ability to load both hydrophilic and hydrophobic drugs and to release the cargo was illustrated. The functionalized ABFs (f-ABFs) can be used to incorporate both hydrophilic and hydrophobic drugs. The preparation of liposome-functionalized microdevices was demonstrated for remotely controlled single-cell drug delivery. These liposomefunctionalized artificial bacterial flagella displayed corkscrew swimming in $3 \mathrm{D}$ with micrometer positioning precision by applying an external rotating magnetic field. The devices are also capable of delivering water-soluble drugs to single cells in vitro (Fig. 7A). ${ }^{43}$ Thermally triggered release of calcein (a common drug analog) from f-ABFs was demonstrated (Fig. 7B). ${ }^{44}$ The fluorescent signals on f-ABFs greatly decreased

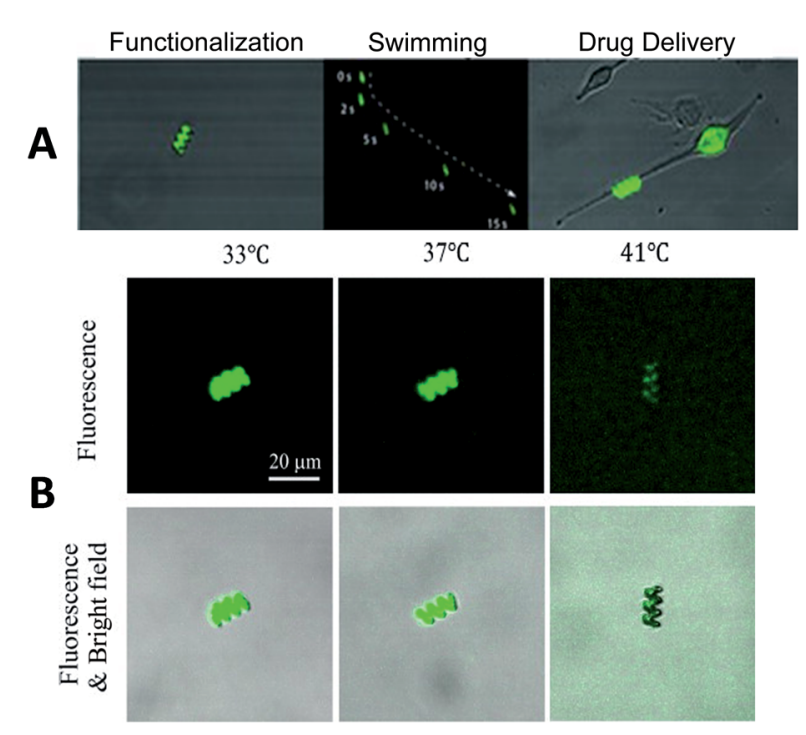

Fig. 7 Magnetically propelled helical microswimmer for drug delivery. (A) f-ABF swimming and calcein delivery to single cells. (a) A representative time-lapse follow-up of the swimming of functionalized artificial bacterial flagella ( $f-A B F$ ) coated with calcein-loaded liposomes. (b) Representative calcein delivery from $\mathrm{f}-\mathrm{ABF}$ to single cells at low magnification (left), high magnification of calcein delivery (middle) and calcein delivered to cells after removing the $\mathrm{f}-\mathrm{ABF}$ (right). (Reproduced from ref. 43, Wiley 2014.) (B) Calcein release from DPPC/ MSPC-functionalized ABFs at 33,37 and $41^{\circ} \mathrm{C}$, respectively. The upper three pictures are fluorescence images, and the lower three pictures are the combined images of fluorescence and bright fields. (Reproduced from ref. 44, Elsevier 2014.) upon increasing the temperature from 37 to $41{ }^{\circ} \mathrm{C}$ and the fluorescence intensity of the background increased accordingly, indicating the significant release of entrapped calcein from the DPPC/monostearoyl phosphatidylcholine (MSPC) liposomes at $41{ }^{\circ} \mathrm{C}$. These f-ABFs offer considerable potential for drug delivery.

Despite the significant progress of the nanomotor design and our new understanding of the ability of magnetic micromotors to transport cargo, several key challenges still exist for their practical in vivo use. While eliminating the fuel requirements, attention must be given also to the preparation of more biocompatible magnetic nanoswimmers. A protective coating around a magnetic nanomotor is essential to prevent etching of the magnetic material by blood components. Cleavable linkers, responsive to tumor microenvironments, are desired to enable an autonomous release of the therapeutic payload to the target site. Significant challenges remain for translating these initial proof-of-concept studies into practical drug delivery applications. Eventually, such magnetically driven fuel-free nanoshuttles are expected to provide an attractive approach for delivering drug cargos to predetermined destinations in a target specific manner.

\section{Ultrasound-powered micro/ nanomotors for drug delivery}

Ultrasound has found extensive applications in medicine, and holds considerable promise for driving micromotors in biological fluids. ${ }^{6,67}$ Recent efforts by the teams of Mallouk's group $^{29,68}$ and our team ${ }^{30,31}$ have illustrated the use of possibility of using ultrasound for propelling gold-nanowire and tubular motors in biologically relevant environments.

Garcia-Gradilla et al. described recently the use of ultrasound-driven nanowire motors based on the nanoporous gold segment for increasing the drug loading capacity. ${ }^{45}$ The new highly porous nanomotors, prepared by dealloying a $\mathrm{Au}-\mathrm{Ag}$ alloy segment, offer a tunable pore size, high surface area, and high capacity for the drug payload. The drug doxorubicin was loaded within the nanopores via electrostatic interactions with an anionic polymeric coating. Ultrasound-driven transport of the loaded drug toward cancer cells was followed by near-
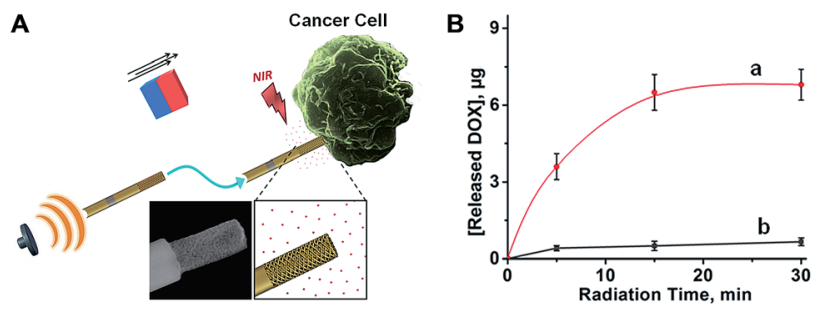

Fig. 8 (A) Schematic of the ultrasound-driven movement of the drugloaded nanoporous Au nanowire motors and triggered release of the drug around a cancer cell. (B) NIR-triggered anticancer drug DOX release as a function of irradiation time using PSS-modified PAu nanomotors (a) and Au nanomotors (b). (Reproduced from ref. 45 , Wiley 2014.) 
infrared light (NIR) triggered release, as shown in Fig. 8. Such a photothermal release has been facilitated by the nanoporous gold structure. Directional movement was achieved by magnetic guidance. The incorporation of the nanoporous gold segment led to a nearly 20 fold increase in the active surface area (compared to common gold nanowires) and to a high loading capacity of $13.4 \mu \mathrm{g} \mathrm{mg}^{-1}$ doxorubicin.

Recently developed ultrasound triggered tubular microbullets are extremely promising for addressing the challenges of limited tissue penetration of therapeutic particles, i.e., directed drug delivery into diseased tissue (Fig. 9A). ${ }^{30}$ Such powerful microbullets, developed by Esener and Wang, ${ }^{30}$ rely on Acoustic Droplet Vaporization (ADV) for propulsion of perfluorocarbonloaded conical-tube microbullets $(\mathrm{MB})$ for penetration into targeted tissue to provide a remarkable force sufficient for penetrating and deforming cellular tissue for potential targeted drug delivery and precision nanosurgery. This highly efficient, powerful and scalable propulsion technique utilizes ultrasound to vaporize biocompatible fuels (i.e., perfluorocarbon PFC emulsions), confined within the interior of a micromachine, for an ultrahigh velocity of over $6 \mathrm{~m} \mathrm{~s}^{-1}$ (i.e., approximately 100 times faster than common micromachines). Such 'bullet-like' propulsion induces sufficient thrust for deep tissue penetration and deformation for potential targeted drug delivery and

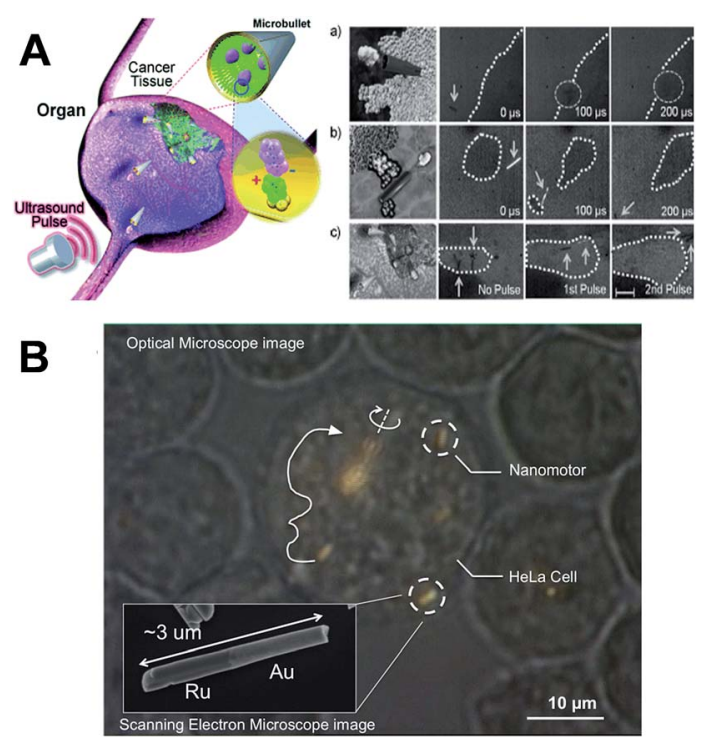

Fig. 9 (A) Computer-aided graphic (graphics on the left) and the corresponding experimental images of PFH-loaded MBs (a) penetrating, (b) cleaving, and (c) expanding tissue following an US pulse signal. All images were taken sequentially at a frame rate of $10000 \mathrm{fps}$ and $10 \times$ objective. US pulses of $44 \mu \mathrm{s} / 1.6 \mathrm{MPa}$ were used for (a) and (b) and short pulses of $4.4 \mu \mathrm{s} / 3.8 \mathrm{MPa}$ were used for (c). Dotted circles and solid arrows are used to indicate the MB's position, while curvilinear dotted lines outline the tissue. (Reproduced from ref. 30, Wiley 2012.) (B) Optical microscope image of a HeLa cell containing several goldruthenium nanomotors. Arrows indicate the trajectories of the nanomotors, and the solid white line shows the propulsion. Near the center of the image, a spindle of several nanomotors is spinning. Inset: electron micrograph of a gold-ruthenium nanomotor. The scattering of sound waves from the two ends results in propulsion. (Reproduced from ref. 68, Wiley 2014). precision nanosurgery. By adjusting the level of propulsion force, different depths in the tissue can be reached. Such a 'bullet-like' carrier must also have the capability of carrying a large therapeutic payload.

The US-triggered microbullet propulsion strategy is expected to have a tremendous impact on diverse biomedical applications, e.g., targeted drug delivery, circulating biolistics, microtissue and artery-cleaning/removal schemes, precision nanosurgery, or cancer surgery. Mallouk's group also demonstrated recently the ultrasonic propulsion of rod-shaped nanomotors inside living HeLa cells (Fig. 9B) ${ }^{68}$ These nanomotors can attach strongly to the external surface of the cells, and are readily internalized by incubation with the cells for periods longer than $24 \mathrm{~h}$. The intracellular propulsion did not involve any chemical fuel and the HeLa cells remained viable. Such developments hold great promise for future in vivo studies of the synthetic nanomotors.

\section{Conclusions}

This review has discussed recent advances in man-made nanomotors towards controlled drug delivery applications. Considerable progress has been made over the past decade in designing a variety of micro/nanomotors for diverse biomedical applications and strategies for the transport and release of therapeutic agents. As our understanding of the design and operation of nanomotors expands, it becomes feasible to utilize the new capabilities of these machines for practical biomedical applications. With increased power and functionality, future micro/nanomachines are expected to perform more demanding biomedical tasks and benefit different drug delivery applications. While key challenges remain prior to applying these nanomotors for in vivo targeted drug delivery, these recent developments advance this objective one step closer to a futuristic nanomachine suitable for improved delivery of therapeutic agents in the body.

Different research teams are currently exploring several routes for realizing future nanomotor-based drug delivery. In order to improve the delivery efficiency, it is essential to explore the attachment of drug carriers to nanomotors through chemical or biological linkers that are sensitive to the tumor microenvironment (e.g., protease enzyme and acidity). Such a mechanism will allow for a more accurate localized drug delivery to the target site using drug-loaded particles powered by external magnetic or ultrasound fields. Improved cellular uptake could be achieved by the incorporation of an appropriate ligand. The combination of multiple functions can lead to more effective operation and this would require the assembly of multiple nano-objects. In particular, incorporating therapeutic or diagnostic entities in the same nanomachine would create theranostic vehicles. Additional efforts should be devoted towards developing high-performance nanomotor locomotion based on in situ fuel sources and alternate powering schemes, offering significant thrust for overcoming the large drag (associated with the presence of the blood cells) and for improving tissue penetration. Future efforts will involve the development of intelligent logic-controlled nanomachines ${ }^{69}$ toward smart 
drug-delivery applications. Further attention should be given to the biocompatibility of drug-delivery nanomotors, and particularly to common metallic constituents such as $\mathrm{Ni}, \mathrm{Ag}$, or Pt. Lastly, the biodegradability (self-destruction) or removal of nanomotors from the body after completing their delivery mission needs further consideration.

The new generation of nanomachine platforms holds even greater promise to improve the treatment of diseases. While the field of nanomachines has been moving at a very rapid pace, such devices have translated gradually into clinical practice. The potential biomedical applications of nanomotors, e.g., nanosurgery or biopsy, extend beyond those discussed in this article and might be limited only by our collective imagination. As research moves toward developing smaller and multifunctional devices, larger multidisciplinary teams, and effective communication between various disciplines, are needed for success. With such collaborative efforts we envision that synthetic nanomotors would provide a new and unique approach to rapidly delivering drug carriers to predetermined destinations. Despite major challenges, the most exciting prospect of nanomachines is the nearly limitless possibilities for the treatment of diseases, towards the realization of Asimov's 1966 microscopic submarine.

\section{Acknowledgements}

This project received support from the Defense Threat Reduction Agency-Joint Science and Technology Office for Chemical and Biological Defense (Grant no. HDTRA1-13-1-0002). W.G. is a HHMI International Student Research fellow.

\section{Notes and references}

1 A. Z. Wang, R. Langer and O. C. Farokhzad, Annu. Rev. Med., 2012, 63, 185.

2 M. E. Davis, Z. G. Chen and D. M. Shin, Nat. Rev. Drug Discovery, 2008, 7, 771.

3 K. Park, ACS Nano, 2013, 7, 7442.

4 T. M. Allen and P. R. Cullis, Science, 2004, 303, 1818.

5 O. M. Koo, I. Rubinstein and H. Onyuksel, Nanomedicine, 2005, 1, 193.

6 O. C. Farokhzad and R. S. Langer, ACS Nano, 2009, 3, 16.

7 S. K. Sahoo and V. Labhasetwar, Drug Discovery Today, 2003, 8, 1112.

8 W. Wang, W. Duan, S. Ahmed, T. E. Mallouk and A. Sen, Nano Today, 2013, 8, 531.

9 J. Wang, Nanomachines: Fundamentals and Applications, Wiley-VCH, Weinheim, Germany, 2013, ISBN 978-3-52733120-8.

10 G. A. Ozin, I. Manners, S. Fournier-Bidoz and A. Arsenault, Adv. Mater., 2005, 17, 3011.

11 J. Wang and W. Gao, ACS Nano, 2012, 6, 5745.

12 B. J. Nelson, I. K. Kaliakatsos and J. J. Abbott, Annu. Rev. Biomed. Eng., 2010, 12, 55.

13 D. Patra, S. Sengupta, W. Duan, H. Zhang, R. Pavlick and A. Sen, Nanoscale, 2013, 5, 1273.
14 L. K. E. A. Abdelmohsen, F. Peng, Y. Tu and D. A. Wilson, J. Mater. Chem. B, 2014, 2, 2395.

15 M. Guix, C. C. Mayorga-Martinez and A. Merkoci, Chem. Rev., 2014, 114, 6285.

16 W. Gao and J. Wang, ACS Nano, 2014, 8, 3170.

17 L. Soler and S. Sanchez, Nanoscale, 2014, 6, 7175.

18 S. Sanchez and M. Pumera, Chem.-Asian J., 2009, 4, 1402.

19 W. F. Paxton, K. C. Kistler, C. C. Olmeda, A. Sen, S. K. St Angelo, Y. Y. Cao, T. E. Mallouk, P. E. Lammert and V. H. Crespi, J. Am. Chem. Soc., 2004, 126, 13424.

20 S. Fournier-Bidoz, A. C. Arsenault, I. Manners and G. A. Ozin, Chem. Commun., 2005, 441.

21 Y. F. Mei, G. S. Huang, A. A. Solovev, E. Bermúdez Ureña, I. Mönch, F. Ding, T. Reindl, R. K. Y. Fu, P. K. Chu and O. G. Schmidt, Adv. Mater., 2008, 20, 4085.

22 Y. F. Mei, A. A. Solovev, S. Sanchez and O. G. Schmidt, Chem. Soc. Rev., 2011, 40, 2109.

23 W. Gao, S. Sattayasamitsathit, J. Orozco and J. Wang, J. Am. Chem. Soc., 2011, 133, 11862.

24 L. Zhang, J. J. Abbott, L. X. Dong, B. E. Kratochvil, D. Bell and B. J. Nelson, Appl. Phys. Lett., 2009, 94, 064107.

25 A. Ghosh and P. Fischer, Nano Lett., 2009, 9, 2243.

26 W. Gao, S. Sattayasamitsathit, K. M. Manesh, D. Weihs and J. Wang, J. Am. Chem. Soc., 2010, 132, 14403.

27 S. Tottori, L. Zhang, F. M. Qiu, K. K. Krawczyk, A. FrancoObregon and B. J. Nelson, Adv. Mater., 2012, 24, 811.

28 W. Gao, X. Feng, A. Pei, C. R. Kane, R. Tam, C. Hennessy and J. Wang, Nano Lett., 2014, 14, 305.

29 W. Wang, L. A. Castro, M. Hoyos and T. E. Mallouk, ACS Nano, 2012, 6, 6122.

30 D. Kagan, M. J. Benchimol, J. C. Claussen, E. ChuluunErdene, S. Esener and J. Wang, Angew. Chem., Int. Ed., 2012, 124, 7637.

31 V. Garcia-Gradilla, J. Orozco, S. Sattayasamitsathit, F. Soto, F. Kuralay, A. Pourazary, A. Katzenberg, W. Gao, Y. Shen and J. Wang, ACS Nano, 2013, 7, 9232.

32 S. Balasubramanian, D. Kagan, C. J. Hu, S. Campuzano, M. J. Lobo-Castañon, N. Lim, D. Y. Kang, M. Zimmerman, L. Zhang and J. Wang, Angew. Chem., Int. Ed., 2011, 50, 4161.

33 D. Kagan, S. Campuzano, S. Salasubramanian, F. Kuralay, G. U. Flechsig and J. Wang, Nano Lett., 2011, 11, 2083.

34 D. Kagan, R. Laocharoensuk, M. Zimmerman, C. Clawson, S. Balasubramanian, D. Kang, D. Bishop, S. Sattayasamitsathit, L. Zhang and J. Wang, Small, 2010, 6, 2741.

35 Y. Wu, Z. Wu, X. Lin, Q. He and J. Li, ACS Nano, 2012, 6, 10910.

36 Z. Wu, Y. Wu, W. He, X. Lin, J. Sun and Q. He, Angew. Chem., Int. Ed., 2013, 52, 7000.

37 M. Xuan, J. Shao, X. Lin, L. Dai and Q. He, ChemPhysChem, 2014, DOI: 10.1002/cphc.201402111.

38 Y. Wu, X. Lin, Z. Wu, H. Mohwald and Q. He, ACS Appl. Mater. Interfaces, 2014, 6, 10476.

39 C. Li, M. Liu, L. Liu, W. Gao, M. Su, Y. Ge, L. Shi, H. Zhang and B. Dong, Nanoscale, 2014, 6, 8601.

40 F. Mou, C. Chen, Q. Zhong, Y. Yin, H. Ma and J. Guan, ACS Appl. Mater. Interfaces, 2014, 6, 9897. 
41 S. Sattayasamitsathit, H. Kou, W. Gao, W. Thavarajah, K. Kaufmann, L. Zhang and J. Wang, Small, 2014, 10, 2830. 42 W. Gao, D. Kagan, O. S. Pak, C. Clawson, S. Campuzano, E. C. Erdene, E. Shipton, E. E. Fullerton, L. Zhang, E. Lauga and J. Wang, Small, 2012, 8, 460.

43 R. Mhanna, F. Qiu, L. Zhang, Y. Ding, K. Sugihara, M. Zenobi-Wong and B. J. Nelson, Small, 2014, 10, 1953.

44 F. Qiu, R. Mhanna, L. Zhang, Y. Ding, S. Fujita and B. J. Nelson, Sens. Actuators, B, 2014, 196, 676.

45 V. Garcia-Gradilla, S. Sattayasamitsathit, F. Soto, F. Kuralay, C. Yardımcı, D. Wiitala, M. Galarnyk and J. Wang, Small, 2014, DOI: 10.1002/smll.201401013.

46 A. A. Solovev, S. Sanchez, M. Pumera, Y. F. Mei and O. G. Schmidt, Adv. Funct. Mater., 2010, 20, 2430.

47 W. Gao, S. Sattayasamitsathit and J. Wang, Chem. Rec., 2012, 12, 224.

48 K. M. Manesh and J. Wang, Small, 2010, 6, 338.

49 J. Wang, Lab Chip, 2012, 12, 1944.

50 A. A. Solovev, W. Xi, D. H. Gracias, S. M. Harazim, C. Deneke, S. Sanchez and O. G. Schmidt, ACS Nano, 2012, 6, 1751.

51 Z. Wu, X. Lin, Y. Wu, T. Si, J. Sun and Q. He, ACS Nano, 2014, 8, 6097.

52 W. Gao, A. Uygun and J. Wang, J. Am. Chem. Soc., 2012, 134, 897.

53 W. Gao, M. D'Agostino, V. Garcia-Gradilla, J. Orozco and J. Wang, Small, 2013, 9, 467.

54 W. Gao, A. Pei and J. Wang, ACS Nano, 2012, 6, 8432.

55 W. Gao, X. Feng, A. Pei, Y. Gu, J. Li and J. Wang, Nanoscale, 2013, 5, 4696.
56 F. Mou, C. Chen, H. Ma, Y. Yin, Q. Wu and J. Guan, Angew. Chem., Int. Ed., 2013, 52, 7208.

57 N. Mano and A. Heller, J. Am. Chem. Soc., 2005, 127, 11574. 58 I. Bozic, J. G. Reiter, B. Allen, T. Antal, K. Chatterjee, P. Shah, Y. S. Moon, A. Yaqubie, N. Kelly, D. T. Le, E. J. Lipson, P. B. Chapman, L. A. Diaz Jr, B. Vogelstein and M. A. Nowak, eLife, 2013, 2, e00747.

59 S. T. Chang, V. N. Paunov, D. N. Petsev and O. D. Velev, Nat. Mater., 2007, 6, 235.

60 G. Loget and A. Kuhn, J. Am. Chem. Soc., 2010, 132, 15918.

61 P. Calvo-Marzal, S. Sattayasamitsathit, S. Balasubramanian, J. R. Windmiller, C. Dao and J. Wang, Chem. Commun., 2010, 46, 1623.

62 M. Liu, T. Zentgraf, Y. M. Liu, G. Bartal and X. Zhang, Nat. Nanotechnol., 2010, 5, 570.

63 P. L. Venugopalan, R. Sai, Y. Chandorkar, B. Basu, S. Shivashankar and A. Ghosh, Nano Lett., 2014, 14, 1968.

64 K. E. Peyer, S. Tottori, F. Qiu, L. Zhang and B. J. Nelson, Chem.-Eur. J., 2013, 19, 28.

65 K. E. Peyer, L. Zhang and B. J. Nelson, Nanoscale, 2013, 5, 1259.

66 H. D. Liang and M. J. K. Blomley, Br. J. Radiol., 2003, 76, S140.

67 W. G. Pitt, G. A. Husseini and B. J. Staples, Expert Opin. Drug Delivery, 2004, 1, 37.

68 W. Wang, S. Li, L. Mair, S. Ahmed, T. J. Huang and T. E. Mallouk, Angew. Chem., Int. Ed., 2014, 53, 3201.

69 S. M. Douglas, I. Bachelet, G. M. Church, S. M. Douglas, I. Bachelet and G. M. Church, Science, 2012, 335, 831. 